\title{
Property of regression estimators in GEE models for ordinal responses
}

\author{
Hyun Yung Lee ${ }^{1}$ \\ ${ }^{1}$ Department of Mathematics Education, Silla University \\ Received 12 November 2011, revised 2 December 2011, accepted 14 December 2011
}

\begin{abstract}
The method of generalized estimating equations (GEEs) provides consistent estimates of the regression parameters in a marginal regression model for longitudinal data, even when the working correlation model is misspecified (Liang and Zeger, 1986). In this paper we compare the estimators of parameters in GEE approach. We consider two aspects: coverage probabilites and efficiency. We adopted to ordinal responses the results derived from binary outcomes.
\end{abstract}

Keywords: Generalized estimating equations, ordinal responses, parameter estimation, repeated measures.

\section{Introduction}

In medical research, disease can be measured with categorical data including a dichotomous, polychotomous or ordinal score for conditions such as the presence or absence of dysplasia or the severity of wheeze. Furthermore, the same observed unit can be assessed by different medical investigators.

Clustered data are frequent in biological and medical experimental research. This includes longitudinal studies, where individuals are observed over time (Agresti, 2002; Diggle et al., 2002) or for other dimensions such as distance to some origin (Singer and Andrade, 1986), and also in family studies (Ziegler et al., 1998; Yan and Fine, 2004). Cho (2010) also recommended about the longitudinal data. In this context, Liang and Zeger (1986) proposed the generalized estimating equations (GEE) approach, which not only models the marginal means in terms of covariates but also incorporates the association between cluster responses. For the case of clustered ordinal data Nores and Diez (2008) investigated some properties of GEE according to working correlation structures. They compared the coverage probability of confidence interval and the efficiency in the sense of variance estimates. The asymptotic efficiency of a correctly specified exchangeable association structure relative to the independence was discussed.

In this paper we study some properties of the estimators of marginal mean parameters in the context of the GEE approach of Heagerty and Zeger (1996) for ordinal data. We focus on two aspects: coverage probabilities and efficiency. For the first one, we made a

\footnotetext{
${ }^{1}$ Full time lecturer, Department of Mathematics Education, Silla University, Pusan 617-736, Korea. E-mail: hylee@silla.ac.kr
} 
simulation study and calculated empirical levels of the confidence intervals for regression parameters based on the sandwich variance estimator. Lipsitz et al. (1991) studied these coverage probabilities in binary responses for a sample size of 100. We considered 100, 120 clusters. Concerning efficiency, some other authors have investigated the loss of efficiency that can occur when assuming different working association structures. However, their studies were confined to continuous or discrete data, principally binary outcomes. Mancl and Leroux (1996) studied asymptotic efficiency by considering an independence working specification in relation to a correctly specified exchangeable association structure. Choi (2010) suggested a mixed-effects model for analyzing split-plot data when there is a repeated measures factor that affects on the response variable. And Choi (2008a, 2008b, 2008c) suggested a marginal probability model for analyzing repeated polytomous response data and binary response data.

This article is organized as follows. Section 2 describes the notations in GEE model for the ordinal responses. In Section 3 we show the simulation schemes used and the results concerning confidence levels. The final section discusses the results obtained.

\section{Notations in GEE model for ordinal responses}

Suppose that a longitudinal study consists of ordinal responses with $(J+1)$ categories and $p$-dimensional covariate vectors $\left(Y_{i t}, x_{i t}\right)$, for $i=1, \cdots, n$ and $t=1, \cdots, n_{i}$, where $Y_{i t}$ denotes the observation for subject $i$ at occasion $t$ and the covariate vector $\boldsymbol{x}_{i t}$ can be discrete or continuous. For simplicity we assume equal occasions, $n_{i}=T$. Denote $Y_{i t}$ as a vector of $J$ indicator variables, $\boldsymbol{y}_{i t}=\left(y_{i t}^{1}, \cdots, y_{i t}^{J}\right)^{\prime}$ with $y_{i t}^{j}=1$ if response $Y_{i t}=j$ and 0 otherwise. Let $\boldsymbol{\pi}_{i t}$ and $\eta_{i t}^{(j)}$ represent the vector of marginal probabilities and the marginal cumulative probabilities, respectively, where $\pi_{i t}=\left(\pi_{i t}^{(1)}, \cdots, \pi_{i t}^{(J)}\right)^{\prime}$ with $\pi_{i t}^{(j)}=$ $P\left(Y_{i t}=j \mid \boldsymbol{x}_{i t}\right)=P\left(Y_{i t}^{(j)}=1 \mid \boldsymbol{x}_{i t}\right)$ and $\eta_{i t}^{(j)}=P\left(Y_{i t} \leq j \mid \boldsymbol{x}_{i t}\right)=\sum_{k=1}^{j} \pi_{i t}^{(k)}$. It can be straightforwardly shown that $E\left(\boldsymbol{y}_{i t}\right)=\pi_{i t}$ and $\operatorname{Var}\left(\boldsymbol{y}_{i t}\right)=\boldsymbol{V}_{i t}=\operatorname{diag}\left(\boldsymbol{\pi}_{i t}\right)-\boldsymbol{\pi}_{i t} \boldsymbol{\pi}_{i t}^{\prime}$. The cumulative logit model with proportional odds assumption for describing the dependence of $Y_{i t}$ on $\boldsymbol{x}_{i t}$ is given by

$$
\operatorname{logit}\left(\eta_{i t}^{(j)}\right)=\log \left(\frac{\eta_{i t}^{(j)}}{1-\eta_{i t}^{(j)}}\right)=\lambda_{j}+\boldsymbol{x}_{i t}^{\prime} \beta, \text { for } j=2, \cdots, J
$$

where the intercepts $\lambda_{1}, \cdots, \lambda_{J}$ satisfy $\lambda_{1} \leq \cdots \leq \lambda_{J}$, is the vector of regression coefficients with $\boldsymbol{\beta}=\left(\beta_{1}, \cdots, \beta_{p}\right)^{\prime}$, and $\boldsymbol{\zeta}_{i t}^{(j)}$ is the $j$ th element of a $J$-dimensional linear predictor $\boldsymbol{\zeta}_{i t}=\left(\zeta_{i t}^{(1)}, \cdots, \zeta_{i t}^{(J)}\right)^{\prime}$. Since $\pi_{i t}^{(1)}=\eta_{i t}^{(1)}$ and

$$
\begin{aligned}
\pi_{i t}^{(j)} & =\eta_{i t}^{(j)}-\eta_{i t}^{(j-1)} \\
& =P\left(Y_{i t}=j \mid \boldsymbol{x}_{i t}\right)-P\left(Y_{i t}=j-1 \mid \boldsymbol{x}_{i t}\right) \\
& =\frac{\exp \left(\eta_{i t}^{(j)}\right)}{1+\exp \left(\eta_{i t}^{(j)}\right)}-\frac{\exp \left(\eta_{i t}^{(j-1)}\right)}{1+\exp \left(\eta_{i t}^{(j-1)}\right)}, \text { for } j=2, \cdots, J
\end{aligned}
$$

the linear predictor $\zeta_{i t}$ can be rewritten as $\boldsymbol{\zeta}_{i t}^{\prime}=\boldsymbol{Z}_{i t}^{\prime} \boldsymbol{\theta}$ with the parameter vector $\boldsymbol{\theta}=$ 
$\left(\lambda_{1}, \cdots, \lambda_{J}, \beta^{\prime}\right)^{\prime}$ and the $J \times(J+p)$ design matrix

$$
\boldsymbol{Z}_{i t}^{\prime}=\left[\begin{array}{llll}
1 & & & \boldsymbol{X}_{i t}^{\prime} \\
& \ddots & & \boldsymbol{X}_{i t}^{\prime} \\
& & 1 & \boldsymbol{X}_{i t}^{\prime}
\end{array}\right]
$$

A $J$-dimensional link function $g$ connects $\pi_{i t}$ and the linear predictor $\boldsymbol{Z}_{i t}^{\prime} \boldsymbol{\theta}$ as $\boldsymbol{\pi}_{i t}=$ $g^{-1}\left(\boldsymbol{Z}_{i t}^{\prime} \boldsymbol{\theta}\right)$. Denote the responses, the marginal probabilities and the design matrix for subject $i$ as $\boldsymbol{Y}_{i}=\left(\boldsymbol{y}_{i 1}^{\prime}, \cdots, \boldsymbol{y}_{i T}^{\prime}\right), \boldsymbol{\pi}_{i}=\left(\pi_{i 1}^{\prime}, \cdots, \pi_{i T}^{\prime}\right)$, and $\boldsymbol{Z}_{i}=\left(\boldsymbol{Z}_{i 1}, \cdots, \boldsymbol{Z}_{i T}\right)_{T J \times(J+P)}^{\prime}$, respectively. The multivariate generalized estimating equations proposed by Lipsitz et al. (1994) and Liang and Zeger (1986) for estimating $\theta$ is the solution to

$$
\sum_{i=1}^{n} \boldsymbol{D}_{i}^{\prime} \boldsymbol{V}_{i}^{\prime^{-1}}\left(\boldsymbol{Y}_{i}-\pi_{i}\right)
$$

where $\boldsymbol{D}_{i}=\partial \boldsymbol{\pi}_{i} / \partial \boldsymbol{\theta}=\left(\boldsymbol{D}_{i 1}^{\prime}, \cdots, D_{i T}^{\prime}\right)^{\prime}$ with the $j$ th row vector of $D_{i t}$ expressed by $\left(\partial \boldsymbol{\pi}_{i t}^{(j)} / \partial \lambda_{1}, \cdots, \partial \boldsymbol{\pi}_{i t}^{(j)} / \partial \beta_{p}\right)^{\prime}$ for $j=1, \cdots, J, t=1, \cdots, T$, and $\boldsymbol{V}_{i}=\boldsymbol{A}_{i}^{1 / 2} \boldsymbol{R}_{i}(\alpha) \boldsymbol{A}_{i}^{1 / 2}$. Here $\boldsymbol{A}_{i}=\operatorname{diag}\left(\boldsymbol{A}_{i 1}, \cdots, \boldsymbol{A}_{i T}\right)$ with diagonal block $\boldsymbol{A}_{i T}=\operatorname{diag}\left(\pi_{i t}^{(1)}\left(1-\pi_{i t}^{(1)}\right), \cdots, \pi_{i t}^{(J)}\left(1-\pi_{i t}^{(J)}\right)\right)$, the "working correlation matrix $\mathbf{R}_{i}(\alpha)$ is the correlation of $\boldsymbol{Y}_{\mathrm{i}}$, and $\alpha$ is a vector of parameters involved in the working correlation structure. In general, $\boldsymbol{V}_{i} \neq \operatorname{Var}\left(\boldsymbol{V}_{i}\right)$. Liang and Zeger (1986) proposed a 'working' correlation matrix to gain efficiency in estimating $\boldsymbol{\theta}$. The solution to (2.2) is a consistent estimate of $\boldsymbol{\theta}$ for a variety of settings of the $T J \times T J$ 'working' correlation matrices. Let $\mathbf{R}_{i}(\alpha)$ be expressed as follows:

$$
\boldsymbol{R}_{i}(\alpha)=\left[\begin{array}{cccc}
\boldsymbol{M}_{i 1} & \boldsymbol{B}_{i 12} & \cdots & \boldsymbol{B}_{i T 1} \\
\boldsymbol{B}_{i 21} & \boldsymbol{M}_{i 2} & \cdots & \boldsymbol{B}_{i T 2} \\
\vdots & \vdots & \ddots & \vdots \\
\boldsymbol{B}_{i T 1} & \boldsymbol{B}_{i T 2} & \cdots & \boldsymbol{M}_{i T}
\end{array}\right]_{T J \times T J}
$$

where the $t^{t h}$ diagonal block $\boldsymbol{M}_{i t}=\boldsymbol{A}_{i t}^{-1 / 2} \boldsymbol{V}_{i t} \boldsymbol{A}_{i t}^{-1 / 2}$ denoted throughout by $\pi_{i}$ for $t=$ $1, \cdots, T$, and the off-diagonal matrix $\boldsymbol{B}_{i s t}=\boldsymbol{A}_{i s}^{-1 / 2} \mathrm{E}\left[\left(\boldsymbol{y}_{i s}-\boldsymbol{\pi}_{i s}\right)\left(\boldsymbol{y}_{i t}-\boldsymbol{\pi}_{i t}\right)^{\prime}\right] \mathbf{A}_{i t}^{-1 / 2}$ parameterized by $\alpha$ for $s \neq t$. The correlations of longitudinal ordinal data include two parts. One is the correlation between the repeated responses for subject $i$ specified by $\mathbf{R}_{\mathrm{i}}(\alpha)$ and the other is the correlation between ordinal categories specified by $\boldsymbol{B}_{\text {ist }}$. The estimation of $\theta$ based on the Fisher scoring algorithm and iterative proportional fitting can be obtained by the geepack package in $\mathrm{R}$ software. Once the estimate of the parameter vector $\hat{\theta}$ is derived, the standardized residual vector, $\widehat{\boldsymbol{e}}_{i}=\left(\widehat{\boldsymbol{e}}_{i 1}^{\prime}, \cdots, \widehat{\boldsymbol{e}}_{i T}\right)^{\prime}$, can be computed with the element vector $\widehat{\boldsymbol{e}}_{i t}=\widehat{\boldsymbol{A}}^{-1 / 2}\left(\boldsymbol{y}_{i t}-\widehat{\boldsymbol{\pi}}_{i t}\right)$, where $\widehat{\boldsymbol{\pi}}_{i t}=g^{-1}\left(\widehat{\boldsymbol{Z}}_{i t}^{\prime} \widehat{\boldsymbol{\theta}}\right)$ for $i=1, \cdots, n ; t=1, \cdots, T$.

In this article, four 'working' correlation matrices, independence $\left(\boldsymbol{R}_{\mathrm{i}}(\alpha)=\boldsymbol{I}\right.$, the identity matrix), $\operatorname{AR}(1)$ structure, exchangeable structure $\left(\boldsymbol{B}_{i s t}=\boldsymbol{B}\right)$ and unspecified structure 
$\left(\boldsymbol{B}_{i s t}=\boldsymbol{B}\right)$, are considered; namely

$$
\begin{gathered}
\boldsymbol{R}_{i n d, i}(\alpha)=\boldsymbol{I}_{T J \times T J}, \boldsymbol{R}_{a r(1), i}(\alpha)=\left[\begin{array}{ccccc}
\boldsymbol{M}_{i 1} & \boldsymbol{L} & \boldsymbol{L}^{2} & \cdots & \boldsymbol{L}^{T-1} \\
L & \boldsymbol{M}_{i 2} & \boldsymbol{L} & \cdots & \boldsymbol{L}^{T-2} \\
\boldsymbol{L}^{2} & \boldsymbol{L} & \boldsymbol{M}_{i 3} & \cdots & L^{T-3} \\
\vdots & \vdots & \vdots & \vdots & \vdots \\
\boldsymbol{L}^{T-1} & \boldsymbol{L}^{T-2} & \boldsymbol{L}^{T-3} & \cdots & \boldsymbol{M}_{i T}
\end{array}\right], \\
\boldsymbol{R}_{e x, i}(\alpha)=\left[\begin{array}{cccc}
\boldsymbol{M}_{i 1} & \boldsymbol{B} & \cdots & \boldsymbol{B} \\
\boldsymbol{B} & \boldsymbol{M}_{i 2} & \cdots & \boldsymbol{B} \\
\vdots & \vdots & \ddots & \vdots \\
\boldsymbol{B} & \boldsymbol{B} & \cdots & \boldsymbol{M}_{i T}
\end{array}\right] \text { and } \boldsymbol{R}_{u n, i}(\alpha)=\left[\begin{array}{cccc}
\boldsymbol{M}_{i 1} & \boldsymbol{B}_{12} & \cdots & \boldsymbol{B}_{1 T} \\
B_{21} & \boldsymbol{M}_{i 2} & \cdots & \boldsymbol{B}_{2 T} \\
\vdots & \vdots & \ddots & \vdots \\
\boldsymbol{B}_{T 1} & \boldsymbol{B}_{T 2} & \cdots & \boldsymbol{M}_{i T}
\end{array}\right]
\end{gathered}
$$

for $i=1, \cdots, n$ as well as the corresponding off-diagonal matrices are estimated by

$$
\begin{array}{r}
\widehat{\boldsymbol{L}}=\frac{\sum_{t=1}^{T-1} \sum_{i=1}^{n} \widehat{e}_{i t} \widehat{e}_{i, t+1}}{(T-1)(n-p)}, \widehat{\boldsymbol{B}}=\frac{\sum_{i=1}^{n} \sum_{s<t} \widehat{e}_{i s} \widehat{e}_{i t}}{\left[\frac{1}{2} \sum_{i=1}^{n} T(T-1)\right]-p}, \\
\widehat{\boldsymbol{B}}_{s t}=\frac{\sum_{i=1}^{n} \widehat{e}_{i s} \widehat{e}_{i t}}{n-p} \text { for } s \neq t=1, \cdots, T .
\end{array}
$$

\section{Coverage probabilities of the regression estimators}

\subsection{Example}

As an illustration, we present an analysis for the dataset from Costa et al. (2006), which consists of 48 patients suffering from rupture of the tendo achilles.

We assume the following GLM relationship

$$
\operatorname{logit}(P[Y \leq j])=\alpha_{j}+\beta_{1} t+\beta_{2} x .
$$

where $t$ is time $(t=1,2,3)$ which is composed of 3 month, 6 month and 1 year and $x$ is treat $(x=1,2$; operativelyand nonoperatively). The response variable is a status of activity $(j=1,2,3)$ those are normal sporting activity, walking, stair climbing and work activity.

\begin{tabular}{ccccc} 
Table 3.1 Estimated parameter estimates and their standard errors \\
\hline \hline Assumed working correlation & $\alpha_{1}$ & $\alpha_{2}$ & $\beta_{1}$ & $\beta_{2}$ \\
\hline \multirow{2}{*}{ Indep } & $0.9980^{1)}$ & -1.5969 & 2.5521 & 3.1640 \\
& $0.7464^{2)}$ & 1.0865 & 1.3157 & 0.7364 \\
\hline \multirow{2}{*}{ Exchangeable } & -4.7937 & -1.6097 & 2.5864 & 3.1386 \\
& 1.1675 & 1.0776 & 1.3003 & 0.7154 \\
\hline \multirow{2}{*}{ AR1 } & -4.7827 & -1.5969 & 2.5521 & 3.1309 \\
& 1.1781 & 1.0865 & 1.3157 & 0.7212 \\
\hline
\end{tabular}

1) GEE estimate 2) Srandard Error (S. E.) of GEE estimate 


\subsection{Simulation schemes}

In order to study empirically the validity of inferences for the regression parameters in the GEE approach of Heagerty and Zeger (1996), we conducted a simulation study. In this section we present the study of the asymptotic efficiency of the estimator $\widehat{\beta}_{I}$, using an independence working specification, relative to $\widehat{\beta}_{E X}$, assuming a correctly specified exchangeable association structure. For each element of $\beta$, the asymptotic relative efficiency (ARE) is given by the ratio of the variance of the regression estimators.

To evaluate the performance of the proposed tests for the proportional odds model fit in terms of type I error rate and the power, the simulated longitudinal ordinal data are generated from the following models:

$$
\text { Model } 1 ; \operatorname{logit}(P[Y \leq j])=\alpha_{j}+\beta_{1} D_{i t}+\beta_{2} x_{i t},
$$

where the monotone difference intercepts are assigned by $\alpha=(-1.0,0,0.5)^{\prime}, \beta_{1}=0, \beta_{2}=$ $-0.1 ; D_{i t}$ equals to 0 for $i \leq n / 2$ and 1 elsewhere, and $x_{i t} \sim U(-1,1)$ for $i=1, \cdots, n$, $t=1,2,3, j=1,2,3$ and $n=(100,120), \rho=(0.5,0.7)$. Here $D_{1 i}$ is time-stationary covariate and $X_{i t}$ is a time-dependent covariate. The pairwise correlations between the observations at the three occasions within a subject are assumed to be 0.5 . The number of repitition is set to be 1,000 .

The simulation study has been implemented through $\mathrm{R}$ software and its library functions. We may refer to a $\mathrm{R}$ Development Team (2006) for the R language and its environment.

\subsection{Simulation results}

The empirical coverage of confidence intervals for $\alpha_{1}, \alpha_{2}, \alpha_{3}, \beta_{1}$ and $\beta_{2}$ are listed in Table 3.2 through Table 3.5 according to the assumed working correlation structure, the sample sizes, the nominal confidence level, and the correlation parameter $\rho$. The empirical coverages attain the nominal confidence levels when $n=(100,120)$. When the true correlation structure is exchangeable with $\rho=0.5$, the length of confidence intervals under the independence working correlation are wider than the others.

The results of exchangeable correlation and AR1 are very similar when the true correlation structure of repeated responses is exchangeable with $\rho=0.5$ or 0.7 . As we see in Table 3.2 through Table 3.3 the lengths of confidence intervals for the exchangeable correlation structure are little shorter than those under AR1 when the true correlation structure is correctly assumed as exchangeable. On the other hand the empirical coverages of AR1 are sometimes better than those of exchangeable working correlation specification. 
Table 3.2 Empirical coverages of confidence limits for $\alpha_{1}, \alpha_{2}, \alpha_{3}, \beta_{1}$, and $\beta_{2}$ among 1000 repetitions when the true correlation structure is exchangeable with $\rho=0$. 5, 0. 7. (a) $\rho=0.5$

\begin{tabular}{|c|c|c|c|c|c|c|c|}
\hline \multirow{2}{*}{$\mathrm{n}$} & \multirow{2}{*}{ Assumed working correlation } & \multirow{2}{*}{$\alpha$} & \multicolumn{5}{|c|}{ Confidence levels } \\
\hline & & & $\alpha_{1}$ & $\alpha_{2}$ & $\alpha_{3}$ & $\bar{\beta}$ & $\beta_{2}$ \\
\hline \multirow{20}{*}{100} & \multirow{6}{*}{ Indep } & \multirow{2}{*}{$90 \%$} & $0.99801)$ & 0.9960 & 0.9970 & 0.8590 & 0.8570 \\
\hline & & & $0.74642)$ & 0.7102 & 0.7319 & 1.0060 & 0.5771 \\
\hline & & \multirow{2}{*}{$95 \%$} & 1.0000 & 0.9980 & 1.0000 & 0.9990 & 0.8570 \\
\hline & & & 0.8893 & 0.8462 & 0.8721 & 1.1990 & 0.6876 \\
\hline & & \multirow{2}{*}{$99 \%$} & 1.0000 & 0.9990 & 1.0000 & 1.0000 & 0.9990 \\
\hline & & & 1.1710 & 1.1140 & 1.1480 & 1.5780 & 0.9051 \\
\hline & \multirow{6}{*}{ Exchangeable } & \multirow{2}{*}{$90 \%$} & 0.9980 & 0.9960 & 0.8570 & 0.9980 & 0.7130 \\
\hline & & & 0.7459 & 0.7100 & 0.7328 & 1.0050 & 0.4643 \\
\hline & & \multirow{2}{*}{$95 \%$} & 1.0000 & 0.9980 & 1.0000 & 0.9990 & 0.9930 \\
\hline & & & 0.8887 & 0.8459 & 0.8731 & 1.1970 & 0.5532 \\
\hline & & \multirow{2}{*}{$99 \%$} & 1.0000 & 0.9990 & 1.0000 & 1.0000 & 0.9980 \\
\hline & & & 1.1700 & 1.1140 & 1.1490 & 1.5760 & 0.7281 \\
\hline & \multirow{6}{*}{ AR1 } & \multirow{2}{*}{$90 \%$} & 0.9980 & 0.8560 & 0.8570 & 0.9980 & 0.7150 \\
\hline & & & 0.7511 & 0.7144 & 0.7352 & 1.0080 & 0.4804 \\
\hline & & \multirow{2}{*}{$95 \%$} & 1.0000 & 0.9980 & 1.0000 & 0.9990 & 0.9960 \\
\hline & & & 0.8949 & 0.8512 & 0.8760 & 1.2010 & 0.5723 \\
\hline & & \multirow{2}{*}{$99 \%$} & 1.0000 & 0.9990 & 1.0000 & 1.0000 & 0.9980 \\
\hline & & & 1.1780 & 1.1200 & 1.1530 & 1.5810 & 0.7534 \\
\hline & \multirow{2}{*}{ Var Ratio } & Indep vs Uniform & 1.0012 & 1.0007 & 0.9977 & 1.0026 & 1.5452 \\
\hline & & AR1vs Uniform & 1.0140 & 1.0120 & 1.0070 & 1.0070 & 1.0710 \\
\hline \multirow{20}{*}{120} & \multirow{6}{*}{ Indep } & \multirow{2}{*}{$90 \%$} & 0.9260 & 0.8750 & 0.9300 & 0.9040 & 0.8360 \\
\hline & & & 0.6832 & 0.6459 & 0.6592 & 0.9182 & 0.5566 \\
\hline & & \multirow{2}{*}{$95 \%$} & 0.9720 & 0.9560 & 0.9740 & 0.9350 & 0.9050 \\
\hline & & & 0.8140 & 0.7696 & 0.7855 & 1.0940 & 0.6632 \\
\hline & & \multirow{2}{*}{$99 \%$} & 1.0000 & 0.9810 & 0.9990 & 0.9600 & 0.9950 \\
\hline & & & 1.0720 & 1.0130 & 1.0340 & 1.4400 & 0.8730 \\
\hline & & \multirow{2}{*}{$90 \%$} & 0.9070 & 0.8940 & 0.9320 & 0.9030 & 0.7540 \\
\hline & & & 0.6841 & 0.6466 & 0.6598 & 0.9190 & 0.4507 \\
\hline & Exchangeable & $95 \%$ & 0.9720 & 0.9560 & 0.9740 & 0.9350 & 0.8450 \\
\hline & & & 0.8151 & 0.7704 & 0.7861 & 1.0950 & 0.5370 \\
\hline & & $99 \%$ & 1.0000 & 0.9990 & 0.9990 & 0.9600 & 0.9920 \\
\hline & & $99 \%$ & 1.0730 & 1.0140 & 1.0350 & 1.4410 & 0.7069 \\
\hline & & $00 \%$ & 0.9450 & 0.8540 & 0.9290 & 0.9040 & 0.8120 \\
\hline & & $90 \%$ & 0.6873 & 0.6491 & 0.6626 & 0.9219 & 0.4779 \\
\hline & $\mathrm{AB} 1$ & $05 \%$ & 0.9720 & 0.9550 & 0.9730 & 0.9540 & 0.8410 \\
\hline & Ant & $90 \%$ & 0.8190 & 0.7734 & 0.7895 & 1.0980 & 0.5694 \\
\hline & & $00 \%$ & 0.9990 & 1.0000 & 0.9980 & 0.9790 & 0.9930 \\
\hline & & $99 \%$ & 1.0780 & 1.0180 & 1.0390 & 1.4460 & 0.7495 \\
\hline & Vor Potio & Indep vs Uniform & 0.9974 & 0.9980 & 0.9983 & 0.9985 & 1.5249 \\
\hline & var katio & AR1vs Uniform & 1.0090 & 1.0080 & 1.0090 & 1.0060 & 1.1240 \\
\hline
\end{tabular}

1) Empirical coverage of confidence interval 2) Length of confidence interval 
Table 3.3 Empirical coverages of confidence limits for $\alpha_{1}, \alpha_{2}, \alpha_{3}, \beta_{1}$ and $\beta_{2}$ among 1000 repetitions when the true correlation structure is exchangeable with $\rho=0.5,0.7$. (b) $\rho=0.7$

\begin{tabular}{|c|c|c|c|c|c|c|c|}
\hline \multirow{2}{*}{$\mathrm{n}$} & \multirow{2}{*}{ Assumed working correlation } & \multirow{2}{*}{$\alpha$} & \multicolumn{5}{|c|}{ Confidence levels } \\
\hline & & & $\alpha_{1}$ & $\alpha_{2}$ & $\alpha_{3}$ & $\beta_{1}$ & $\beta_{2}$ \\
\hline \multirow{20}{*}{100} & \multirow{6}{*}{ Indep } & \multirow{2}{*}{$90 \%$} & $0.96201)$ & 0.9680 & 0.8750 & 0.9630 & 0.7490 \\
\hline & & & $0.81532)$ & 0.7738 & 0.7941 & 1.0980 & 0.5846 \\
\hline & & \multirow{2}{*}{$95 \%$} & 0.9840 & 0.9780 & 0.8930 & 0.9790 & 0.8670 \\
\hline & & & 0.9714 & 0.9220 & 0.9492 & 1.3080 & 0.6966 \\
\hline & & \multirow{2}{*}{$99 \%$} & 0.9960 & 0.9940 & 0.9960 & 0.9900 & 0.8950 \\
\hline & & & 1.2790 & 1.2140 & 1.2460 & 1.7220 & 0.9169 \\
\hline & \multirow{6}{*}{ Exchangeable } & \multirow{2}{*}{$90 \%$} & 0.9630 & 0.8770 & 0.8760 & 0.9620 & 0.5420 \\
\hline & & & 0.8167 & 0.7752 & 0.7968 & 1.0990 & 0.3796 \\
\hline & & \multirow{2}{*}{$95 \%$} & 0.9830 & 0.9790 & 0.8920 & 0.9780 & 0.8500 \\
\hline & & & 0.9731 & 0.9237 & 0.9493 & 1.3090 & 0.4522 \\
\hline & & \multirow{2}{*}{$99 \%$} & 0.9970 & 0.9940 & 0.9960 & 0.9890 & 0.8920 \\
\hline & & & 1.2810 & 1.2160 & 1.2500 & 1.7230 & 0.5953 \\
\hline & \multirow{6}{*}{ AR1 } & \multirow{2}{*}{$90 \%$} & 0.9650 & 0.8760 & 0.8780 & 0.9610 & 0.7310 \\
\hline & & & 0.8229 & 0.7797 & 0.7995 & 1.1030 & 0.4112 \\
\hline & & \multirow{2}{*}{$95 \%$} & 0.9850 & 0.9790 & 0.8910 & 0.9770 & 0.8530 \\
\hline & & & 0.9805 & 0.9290 & 0.9526 & 1.3140 & 0.4900 \\
\hline & & \multirow{2}{*}{$99 \%$} & 0.9970 & 0.9950 & 0.9960 & 0.9900 & 0.9770 \\
\hline & & & 1.2910 & 1.2230 & 1.2540 & 1.7290 & 0.6450 \\
\hline & \multirow{2}{*}{ Var Ratio } & Indep vs Uniform & 0.9964 & 0.9963 & 0.9934 & 0.9988 & 2.3724 \\
\hline & & AR1vs Uniform & 1.0150 & 1.0120 & 1.0070 & 1.0070 & 1.1740 \\
\hline \multirow{20}{*}{120} & \multirow{6}{*}{ Exchangeable } & \multirow{2}{*}{$90 \%$} & 0.9550 & 0.9550 & 0.8870 & 0.9080 & 0.8190 \\
\hline & & & 0.7487 & 0.7034 & 0.7244 & 1.0070 & 0.5569 \\
\hline & & \multirow{2}{*}{$95 \%$} & 0.9780 & 0.9770 & 0.9330 & 0.9310 & 0.8880 \\
\hline & & & 0.8920 & 0.8381 & 0.8630 & 1.1990 & 0.6635 \\
\hline & & \multirow{2}{*}{$99 \%$} & 1.0000 & 1.0000 & 0.9770 & 0.9770 & 0.9780 \\
\hline & & & 1.1740 & 1.1030 & 1.1360 & 1.5790 & 0.8734 \\
\hline & & \multirow{2}{*}{$90 \%$} & 0.9550 & 0.9100 & 0.8870 & 0.9080 & 0.6840 \\
\hline & & & 0.7496 & 0.7047 & 0.7258 & 1.0080 & 0.3656 \\
\hline & Uniform & $95 \%$ & 0.9780 & 0.9770 & 0.9330 & 0.9310 & 0.7530 \\
\hline & & & 0.8931 & 0.8396 & 0.8648 & 1.2010 & 0.4356 \\
\hline & & $09 \%$ & 1.0000 & 1.0000 & 0.9770 & 0.9770 & 0.9780 \\
\hline & & $99 \%$ & 1.1760 & 1.1050 & 1.1380 & 1.5810 & 0.5734 \\
\hline & & $90 \%$ & 0.9550 & 0.9100 & 0.8870 & 0.8850 & 0.7750 \\
\hline & & $90 \%$ & 0.7560 & 0.7095 & 0.7300 & 1.0120 & 0.3981 \\
\hline & $\mathrm{AB} 1$ & $95 \%$ & 0.9780 & 1.0000 & 0.9330 & 0.9540 & 0.8210 \\
\hline & & & 0.9008 & 0.8453 & 0.8698 & 1.2060 & 0.4743 \\
\hline & & $99 \%$ & 1.0000 & 1.0000 & 1.0000 & 0.9770 & 0.9560 \\
\hline & & $99 \%$ & 1.1860 & 1.1130 & 1.1450 & 1.5870 & 0.6243 \\
\hline & Var Batio & Indep vs Uniform & 0.9976 & 0.9963 & 0.9959 & 0.9973 & 2.3197 \\
\hline & val hatio & AR1vs Uniform & 1.0170 & 1.0140 & 1.0120 & 1.0080 & 1.1850 \\
\hline
\end{tabular}

1) Empirical coverage of confidence interval 2) Length of confidence interval 
Table 3.4 Empirical coverages of confidence limits for $\alpha_{1}, \alpha_{2}, \alpha_{3}, \beta_{1}$ and $\beta_{2}$ among 1000 repetitions when the true correlation structure is $\operatorname{AR} 1$ with $\rho=0.5,0.7$. (a) $\rho=0.5$

\begin{tabular}{|c|c|c|c|c|c|c|c|}
\hline \multirow{2}{*}{$\mathrm{n}$} & \multirow{2}{*}{ Assumed working correlation } & \multirow{2}{*}{$\alpha$} & \multicolumn{5}{|c|}{ Confidence levels } \\
\hline & & & $\alpha_{1}$ & $\alpha_{2}$ & $\alpha_{3}$ & $\beta_{1}$ & $\beta_{2}$ \\
\hline \multirow{20}{*}{100} & \multirow{6}{*}{ Indep } & \multirow{2}{*}{$90 \%$} & $1.00001)$ & 1.0000 & 0.8570 & 0.8570 & 0.5710 \\
\hline & & & $0.71502)$ & 0.6852 & 0.6949 & 0.9583 & 0.5722 \\
\hline & & \multirow{2}{*}{$95 \%$} & 1.0000 & 1.0000 & 1.0000 & 1.0000 & 0.8570 \\
\hline & & & 0.8519 & 0.8165 & 0.8280 & 1.1420 & 0.6818 \\
\hline & & \multirow{2}{*}{$99 \%$} & 1.0000 & 1.0000 & 1.0000 & 1.0000 & 1.0000 \\
\hline & & & 1.1210 & 1.0750 & 1.0900 & 1.5030 & 0.8974 \\
\hline & \multirow{6}{*}{ Exchangeable } & \multirow{2}{*}{$90 \%$} & 1.0000 & 0.8570 & 0.8570 & 0.8570 & 0.5710 \\
\hline & & & 0.7144 & 0.6846 & 0.6955 & 0.9566 & 0.4932 \\
\hline & & \multirow{2}{*}{$95 \%$} & 1.0000 & 1.0000 & 1.0000 & 1.0000 & 1.0000 \\
\hline & & & 0.8512 & 0.8157 & 0.8287 & 1.1400 & 0.5877 \\
\hline & & \multirow{2}{*}{$99 \%$} & 1.0000 & 1.0000 & 1.0000 & 1.0000 & 1.0000 \\
\hline & & & 1.1200 & 1.0740 & 1.0910 & 1.5000 & 0.7736 \\
\hline & \multirow{6}{*}{ AR1 } & \multirow{2}{*}{$90 \%$} & 1.0000 & 0.8570 & 0.8570 & 0.8570 & 0.5710 \\
\hline & & & 0.7127 & 0.6816 & 0.6917 & 0.9480 & 0.4674 \\
\hline & & \multirow{2}{*}{$95 \%$} & 1.0000 & 1.0000 & 0.8570 & 1.0000 & 1.0000 \\
\hline & & & 0.8491 & 0.8121 & 0.8241 & 1.1300 & 0.5569 \\
\hline & & \multirow{2}{*}{$99 \%$} & 1.0000 & 1.0000 & 1.0000 & 1.0000 & 1.0000 \\
\hline & & & 1.1180 & 1.0690 & 1.0850 & 1.4870 & 0.7331 \\
\hline & \multirow{2}{*}{ Var Ratio } & Indep vs Uniform & 1.0070 & 1.0110 & 1.0090 & 1.0220 & 1.4990 \\
\hline & & AR1vs Uniform & 1.0050 & 1.0090 & 1.0110 & 1.0180 & 1.1140 \\
\hline \multirow{20}{*}{120} & \multirow{6}{*}{ Indep } & \multirow{2}{*}{$90 \%$} & 0.8690 & 0.8920 & 0.8480 & 0.9040 & 0.8580 \\
\hline & & & 0.6570 & 0.6229 & 0.6353 & 0.8800 & 0.5516 \\
\hline & & \multirow{2}{*}{$95 \%$} & 0.9020 & 0.9250 & 0.9250 & 0.9370 & 0.9010 \\
\hline & & & 0.7828 & 0.7422 & 0.7569 & 1.0490 & 0.6572 \\
\hline & & \multirow{2}{*}{$99 \%$} & 0.9790 & 0.9680 & 0.9780 & 0.9900 & 0.9890 \\
\hline & & & 1.0300 & 0.9769 & 0.9963 & 1.3800 & 0.8651 \\
\hline & & \multirow{2}{*}{$90 \%$} & 0.8690 & 0.8920 & 0.8370 & 0.9040 & 0.8580 \\
\hline & & & 0.6570 & 0.6231 & 0.6358 & 0.8806 & 0.4790 \\
\hline & Fxchanoreable & $95 \%$ & 0.9020 & 0.9250 & 0.9250 & 0.9260 & 0.9020 \\
\hline & & $95 \%$ & 0.7828 & 0.7424 & 0.7575 & 1.0490 & 0.5708 \\
\hline & & $09 \%$ & 0.9790 & 0.9680 & 0.9890 & 0.9900 & 0.9680 \\
\hline & & $99 \%$ & 1.0300 & 0.9773 & 0.9972 & 1.3810 & 0.7513 \\
\hline & & $90 \%$ & 0.8690 & 0.8920 & 0.8700 & 0.9040 & 0.8490 \\
\hline & & $90 \%$ & 0.6523 & 0.6182 & 0.6308 & 0.8727 & 0.4650 \\
\hline & $\mathrm{AB} 1$ & $95 \%$ & 0.9130 & 0.9250 & 0.9140 & 0.9260 & 0.9020 \\
\hline & $\operatorname{An} 1$ & 9070 & 0.7772 & 0.7366 & 0.7516 & 1.0400 & 0.5540 \\
\hline & & $00 \%$ & 0.9790 & 0.9780 & 0.9890 & 0.9900 & 0.9780 \\
\hline & & $99 \%$ & 1.0230 & 0.9696 & 0.9893 & 1.3690 & 0.7293 \\
\hline & Var Batio & Indep vs Uniform & 1.0140 & 1.0150 & 1.0140 & 1.0170 & 1.4070 \\
\hline & val hatio & AR1vs Uniform & 1.0140 & 1.0160 & 1.0160 & 1.0180 & 1.0610 \\
\hline
\end{tabular}

1) Empirical coverage of confidence interval 2) Length of confidence interval 
Table 3.5 Empirical coverages of confidence limits for $\alpha_{1}, \alpha_{2}, \alpha_{3}, \beta_{1}$ and $\beta_{2}$ among 1000 repetitions when the true correlation structure is AR1 with $\rho=0.5,0.7$. (b) $\rho=0.7$

\begin{tabular}{|c|c|c|c|c|c|c|c|}
\hline \multirow{2}{*}{$\mathrm{n}$} & \multirow{2}{*}{ Assumed working correlation } & \multirow{2}{*}{$\alpha$} & \multicolumn{5}{|c|}{ Confidence levels } \\
\hline & & & $\alpha_{1}$ & $\alpha_{2}$ & $\alpha_{3}$ & $\beta_{1}$ & $\beta_{2}$ \\
\hline \multirow{20}{*}{100} & \multirow{6}{*}{ Indep } & \multirow{2}{*}{$90 \%$} & $1.00001)$ & 0.8570 & 0.8570 & 0.8570 & 0.7140 \\
\hline & & & $0.77872)$ & 0.7531 & 0.7703 & 1.0600 & 0.5761 \\
\hline & & \multirow{2}{*}{$95 \%$} & 1.0000 & 1.0000 & 0.8570 & 1.0000 & 0.8570 \\
\hline & & & 0.9278 & 0.8974 & 0.9178 & 1.2630 & 0.6864 \\
\hline & & \multirow{2}{*}{$99 \%$} & 1.0000 & 1.0000 & 1.0000 & 1.0000 & 1.0000 \\
\hline & & & 1.2210 & 1.1810 & 1.2080 & 1.6620 & 0.9035 \\
\hline & \multirow{6}{*}{ Exchangeable } & \multirow{2}{*}{$90 \%$} & 1.0000 & 0.8570 & 0.8570 & 0.8570 & 0.7140 \\
\hline & & & 0.7791 & 0.7533 & 0.7720 & 1.0590 & 0.4124 \\
\hline & & \multirow{2}{*}{$95 \%$} & 1.0000 & 1.0000 & 0.8570 & 1.0000 & 0.8570 \\
\hline & & & 0.9282 & 0.8976 & 0.9198 & 1.2620 & 0.4914 \\
\hline & & \multirow{2}{*}{$99 \%$} & 1.0000 & 1.0000 & 1.0000 & 1.0000 & 1.0000 \\
\hline & & & 1.2220 & 1.1820 & 1.2110 & 1.6610 & 0.6469 \\
\hline & \multirow{6}{*}{$\mathrm{AR} 1$} & \multirow{2}{*}{$90 \%$} & 1.0000 & 0.8570 & 0.8570 & 0.8570 & 0.7140 \\
\hline & & & 0.7781 & 0.7497 & 0.7647 & 1.0480 & 0.3770 \\
\hline & & \multirow{2}{*}{$95 \%$} & 1.0000 & 0.8570 & 0.8570 & 1.0000 & 0.7140 \\
\hline & & & 0.9271 & 0.8933 & 0.9112 & 1.2490 & 0.4492 \\
\hline & & \multirow{2}{*}{$99 \%$} & 1.0000 & 1.0000 & 1.0000 & 1.0000 & 1.0000 \\
\hline & & & 1.2200 & 1.1760 & 1.1990 & 1.6440 & 0.5913 \\
\hline & \multirow{2}{*}{ Var Ratio } & Indep vs Uniform & 1.0020 & 1.0090 & 1.0150 & 1.0210 & 2.3350 \\
\hline & & AR1vs Uniform & 1.0020 & 1.0100 & 1.0190 & 1.0200 & 1.1970 \\
\hline \multirow{20}{*}{120} & \multirow{6}{*}{ Indep } & \multirow{2}{*}{$90 \%$} & 0.8630 & 0.8880 & 0.8610 & 0.8710 & 0.8460 \\
\hline & & & 0.7285 & 0.6903 & 0.7051 & 0.9764 & 0.5528 \\
\hline & & \multirow{2}{*}{$95 \%$} & 0.9160 & 0.9240 & 0.9230 & 0.9240 & 0.9220 \\
\hline & & & 0.8680 & 0.8225 & 0.8401 & 1.1630 & 0.6587 \\
\hline & & \multirow{2}{*}{$99 \%$} & 0.9880 & 0.9770 & 0.9880 & 1.0000 & 0.9870 \\
\hline & & & 1.1430 & 1.0830 & 1.1060 & 1.5310 & 0.8670 \\
\hline & & \multirow{2}{*}{$90 \%$} & 0.8640 & 0.8890 & 0.8510 & 0.8710 & 0.8680 \\
\hline & & & 0.7290 & 0.6911 & 0.7060 & 0.9775 & 0.3980 \\
\hline & & & 0.9160 & 0.9250 & 0.9230 & 0.9230 & 0.9220 \\
\hline & Exchangeable & $95 \%$ & 0.8686 & 0.8234 & 0.8412 & 1.1650 & 0.4742 \\
\hline & & 0007 & 0.9880 & 0.9770 & 0.9880 & 1.0000 & 0.9670 \\
\hline & & $99 \%$ & 1.1430 & 1.0840 & 1.1070 & 1.5330 & 0.6242 \\
\hline & & $00 \%$ & 0.8720 & 0.8880 & 0.8620 & 0.8720 & 0.7930 \\
\hline & & $90 \%$ & 0.7238 & 0.6857 & 0.7010 & 0.9687 & 0.3776 \\
\hline & $\mathrm{AB} 1$ & $95 \%$ & 0.9060 & 0.9340 & 0.9330 & 0.9240 & 0.9220 \\
\hline & AR 1 & $95 \%$ & 0.8624 & 0.8170 & 0.8352 & 1.1540 & 0.4499 \\
\hline & & $09 \%$ & 0.9880 & 0.9670 & 0.9880 & 0.9990 & 0.9780 \\
\hline & & $99 \%$ & 1.1350 & 1.0750 & 1.0990 & 1.5190 & 0.5922 \\
\hline & & Indep vs Uniform & 1.0130 & 1.0140 & 1.0120 & 1.0160 & 2.1430 \\
\hline & Ratio & AR1vs Uniform & 1.0140 & 1.0160 & 1.0140 & 1.0180 & 1.1110 \\
\hline
\end{tabular}

1) Empirical coverage of confidence interval 2) Length of confidence interval

\section{Concluding remarks}

In this article we studied some properties of the estimators of regression parameters in the GEE approach of Heagerty and Zeger (1996) for clustered ordinal data. We focused on this alternative since it includes specific models for ordinal data, both for marginal means and for association between responses. Furthermore, it uses centered variables $y_{i}^{*}-p_{i}$ in the estimating equation for $\alpha$ instead of $y_{i}^{*}$, as proposed by Williamson et al. (1995), which results in estimators that are more efficient and invariant to the codification of the 
response in the case of binary data (Heagerty and Zeger, 1996). We studied asymptotic efficiency of the independence, (Exchangeable, AR1) estimator $\widehat{\beta}_{I},\left(\widehat{\beta}_{E x}, \widehat{\beta}_{A R 1}\right)$ relative to the exchangeable estimator $\widehat{\beta}_{E x}$, when the true association structure is exchangeable. And we studied asymptotic efficiency of the independence, (Exchangeable, AR1) estimator $\widehat{\beta}_{I}$, $\left(\widehat{\beta}_{E x}, \widehat{\beta}_{A R 1}\right)$ relative to the AR1 estimator $\widehat{\beta}_{A R 1}$, when the true association structure is AR1. When the variance ratio is larger than 1 , the true association structure is better than the fitted association structure. Because in the majority of cases the variance ratio of the regression estimates of the GEE model is larger than 1, the exchangeable association structure is recommended. But in the simulation design the case of the repeated time $T=4$ was not considered owing to the running time of simulation. And the case of unspecified working correlation matrix was not considered because it requires so many parameters.

\section{References}

Agresti, A. (2002). Categorical data analysis, 2nd ed., Wiley, New York.

Cho, D. H. (2010). Mixed-effects LS-SVR for longitudinal data. Journal of the Korean Data ES Information Science Society, 21, 363-369.

Choi, J. (2008a). A logit model for repeated binary response data. The Korean Journal of Applied Statistics, 21, 291-299.

Choi, J. (2008b). A marginal logit mixed-effects model for repeated binary response data. Journal of the Korean Data \& Information Science Society, 19, 413-420.

Choi, J (2008c). A marginal probability model for repeated polytomous response data. Journal of the Korean Data \& Information Science Society, 19, 577-585.

Choi, J. (2010). A mixed model for repeated split-plot data. Journal of the Korean Data \&3 Information Science Society, 21, 1-9.

Diggle, P. J., Heagerty, P. J., Liang, K. Y. and Zeger, S. L. (2002). Analysis of longitudinal data, 2nd ed., Oxford University Press, Oxford.

Heagerty, P. J. and Zeger, S. L. (1996). Marginal regression models for clustered ordinal measurements. Journal of the American Statistical Association, 91, 1024-1036.

Liang, K. Y. and Zeger, S. L. (1986). Longitudinal data analysis using generalized linear models. Biometrika, 73, 13-22.

Lipsitz, S. R., Laird, N. M. and Harrington, D. P. (1991). Generalized estimating equations for correlated binary data: Using the odds ratio as a measure of association. Biometrika, 78, 153-60.

Mancl, L. A. and Leroux, B. G. (1996). Efficiency of regression estimates for clusterd data. Biometrics, 52, 500-511.

Nores, M. L. and Diez, M. P. (2008). Some properties of regression estimators in GEE models for clustered ordinal data. Computational Statistics \& 8 Data Analysis, 52, 3877-3888.

R Development Team (2006). R: A language and environment for statistical computing, $\mathrm{R}$ Foundation for Statistical Computing, Vienna, Austria. ISBN 3-900051-07-0, URL:http://www.r-project.org.

Singer, J. M. and Andrade, D. F. (1986). Analise de dados longitudinais. Associacao Brasileira de Estatistica, Sao Paulo, 106.

Yan, J. and Fine, J. (2004). Estimating equations for association structures. Statistic in Medicine, 23, 859-874.

Ziegler, A., Kastner, C. and Blettner, M. (1998). The generalised estimating equations: An annotated bibliography. Biometrical Journal, 40, 115-139. 